\title{
Dominating Set Based Clustering Algorithm for Mobile Adhoc Network
}

\begin{abstract}
A group of mobile nodes using a common wireless media forms a dynamic multihop network known as Mobile Ad-Hoc network (MANET). Creating substructure (cluster) of the mobile adhoc network, basically divides the network into sub network. It has been observed that clustering of large size MANET improves the system performance to a large extent. Clustering allows the MANET to organize it in some hierarchy which reduces control overhead and make it scalable. This paper, gives a new scheme of clustering the MANET that uses the concept of dominating set. The cluster so formed by the proposed method is more stable (less mobile) than the earlier existing algorithms. Here the dominating nodes will be considered as the cluster head of a particular cluster. The non dominating nodes will select the most qualified dominating node as their cluster head and will join the cluster. The quality of the node is measured by its clustering co efficient.
\end{abstract}

A. K. Das , B. Kalita
Index Terms-Mobile Adhoc Network (MANET), Connected Dominating Set (CDS), Unit Disk Graph (UDG), Clustering Co efficient

\section{I.INTRODUCTION}

A Mobile Adhoc Network is an infrastrctureless wireless communication network that support mobile users. In MANET, a number of mobile hosts share a multihop wireless communication channel to form an autonomous system. In MANET, there is no centralized administration and basically it is constructed by self organization of the mobile nodes. The mobile nodes in the MANET can transmit, receive and relay data within the network. The mobile nodes in the network establish routing path among themselves dynamically and hence they are also called infra structure less network.

The nodes in MANET can talk or communicate among themselves only when they are within the transmission range of each other. If nodes are not within each other transmission area, they can still communicate provided other nodes lying in between them relay packets for them.Therefore in multi hop routing, participation of all nodes ensures that the nodes can be reached out.Due to this multihop nature, the same packet may be replicated resulting in uncontrolled flooding which consumes a lot of network bandwidth and increases processing complexity.Moreover the battery power of the nodes are also wasted. To overcome these problems,a number of procedures is proposed to divide the network into clusters. The whole process is termed as clustering which is basically a method of forming similar virtual group of the MANET
The routing and topology management of MANET can be done in a more efficient way by using the concept of clustering. It is seen that with this approach, the performance of the MANET having a large number of nodes has improved.A number of advantages are there of clustering a MANET. One of the advantage is that the resources can be reused and as a result the system capacity also increases. Moreover if we can form cluster that does not overlap, then the same code or frequency can be used by two cluster if they are not neighbouring cluster. Another advantage of cluster formation is that the transmission of data can be co ordinated in a better way with the help of cluster head which is a special node in the cluster. In inter cluster routing, the routing information is confined within the cluster head and gateway node, thus restricting the flow of routing information. Besides this, clustering helps in maintaining a stable MANET for a long period of time. Since the mobile nodes may change its cluster because of dynamic topology, only mobile node in the corresponding cluster needs has to update the information. Thus, any change in routing information need not be updated by the entire network, thus reducing the information processing by each mobile node.

Thus it is seen that there are a lot of benefits of clustering a MANET. Now, the question is how to cluster the MANET so that overall integrity of the network is not compromised. Many approaches have been proposed to cope with the limitations of mobility and battery dependency of the mobile nodes .A lot of clustering approaches is proposed to make the MANET fault tolerant A number of algorithms are proposed to minimize the communication cost and to make it a more stable network. Some methods were also proposed to select the cluster head of a particular cluster using entropy which is the average information. Some authors also propose formation of cluster using mobile agent.

This paper is organized as follows: In section I, introduction about mobile adhoc network is presented with its challenges and the benefits of clustering in routing. In section II, some related works have been studied about the existing clustering algorithms on mobile adhoc network .The definitions of different concepts used in this paper is given in section III. In section IV, we propose a new clustering algorithm in a mobile adhoc network. In section $\mathrm{V}$, implementation of our algorithm in an unit disk graph of 12 vertices has been discussed .In section VI, we proposed a new method for selecting the gateway node. Finally in section VII, we have given the conclusion and future scope of this work.

B Kalita Dept. of Computer Applications, Assam Don Bosco University, Guwahati-781017, Assam, India

E mail: bichitra1_kalita@rediffmail.com 


\section{Dominating Set Based Clustering Algorithm for Mobile Adhoc Network}

\section{RELATED WORK}

In this section we have explored the various works on creating substructure (Cluster) of the mobile adhoc network. A number of methods were proposed by various researchers on clustering a mobile adhoc network. M. Gerla, J. T. C. Tsai [1] proposed a method in which the node which is adjacent to the maximum number of nodes or the node whose degree is maximum is selected as the cluster head node. But one problem with this approach is that since the topology changes frequently, so cluster head will not remain as cluster head for a long period of time. Another approach proposed by A. Ephremides, J. E. Wieselthier, D. J. Baker [2] uses the minimum identifier node as the cluster head but this approach produces a large number of clusters. D. J. Baker, A. Ephremides [3] proposed a linked cluster algorithm in which the cluster head selection is done on the basis of highest id among all nodes. S. Basagni[4 ] [5] proposed a distributed algorithm where the cluster head is selected based on the weights of the node and any tie between node is broken by its ID.But here how the weights are assigned is not mentioned clearly. X. Niu, Z. Tao, G. Wu, C. Huang, L. Cui [6] proposed a hybrid cluster routing algorithm, where cluster head is selected based on the priority assigned by the node In this method, each node maintains a cluster head priority. This method restricts the cluster head change than other methods .In [7] J.Y. Yu, P. H. Joo Chong proposed a quick Efficient Clustering Scheme (ECS) where the selection of cluster head is done on the basis of who delivers the message first to its's neighbours. In WCA [8], various parameters are used for the selection of cluster head. The weight of the node is calculated based on its degree, transmission range and battery power. It works better than other methods but the problem is that the power gets depleted in calculating the weights before the actual clustering process starts .In [9], Z. El-Bazzal, M. Kadoch, B. L. Agba, F. Gagnon proposed an efficient management algorithm for clustering that uses the parameters like degree of a node, remaining battery power, transmission range and node mobility for selecting the cluster head.. Note that in MANETs nodes may do not aware from their location. As discussed above, the main objective of the previous works is to select a node as the $\mathrm{CH}$ of each cluster. Even though this minimizes the overall signaling overhead in the cluster, it mainly can make the central $\mathrm{CH}$ a bottleneck. In [10] multiple cluster heads is used one for each neighboring cluster. By distributing the load, a single $\mathrm{CH}$ does not have to bear all the added responsibility of being the central point for routing in a cluster. The proposed mechanism does not mandate a specific $\mathrm{CH}$ election process.

The following table (Table 1) briefly compares the different clustering algorithm and outlines their benefits and drawbacks.

\begin{tabular}{|c|c|c|c|c|}
\hline $\begin{array}{l}\text { Serial } \\
\text { No. }\end{array}$ & $\begin{array}{l}\text { Clustering } \\
\text { Scheme }\end{array}$ & $\begin{array}{l}\text { Cluster } \\
\text { head } \\
\text { Selection } \\
\text { Criteria }\end{array}$ & Benefits & Drawbacks \\
\hline 1 & Lowest Id & $\begin{array}{l}\text { Node with } \\
\text { minimum } \\
\text { ID which is } \\
\text { distinct. }\end{array}$ & $\begin{array}{l}\text { Simple to } \\
\text { implement. }\end{array}$ & $\begin{array}{l}\text { 1> Cluster } \\
\text { head's } \\
\text { power gets } \\
\text { depleted } \\
\text { quickly due } \\
\text { to increase } \\
\text { overload.. } \\
\text { 2>A } \\
\text { number of } \\
\text { cluster head } \\
\text { is generated }\end{array}$ \\
\hline 2 & $\begin{array}{l}\text { Highest } \\
\text { Degree }\end{array}$ & $\begin{array}{l}\text { Node with } \\
\text { Highest } \\
\text { connectivity } \\
\text { value } \\
\text { among its } \\
\text { direct } \\
\text { neighbours. }\end{array}$ & $\begin{array}{l}\text { Less hop to } \\
\text { fulfil a } \\
\text { request. }\end{array}$ & $\begin{array}{l}\text { 1> Cluster } \\
\text { head gets } \\
\text { congested } \\
\text { due to } \\
\text { dynamic } \\
\text { toplogy } \\
\text { change of } \\
\text { the } \\
\text { MANET.. } \\
\text { 2> A node } \\
\text { is linked to } \\
\text { multiple } \\
\text { nodes. } \\
\text { 3> Single } \\
\text { hop } \\
\text { connection. } \\
\text { 4> One } \\
\text { cluster can } \\
\text { be } \\
\text { exhausted } \\
\text { serving too } \\
\text { many hosts. }\end{array}$ \\
\hline
\end{tabular}

\section{III .BASIC CONCEPTS}

\section{III.i Unit Disk Graph:}

Unit disk graph as shown in Fig 1 is a 2-dimentional plane graph that for $u, v € V$, there is a bidirectional edge between $u$ and $v$, if and only if $\operatorname{Eudist}(u, v) \leq 1$. Here, $\operatorname{Eudist}(u, v)$ is the Euclidean Distance between $u$ and $v$.

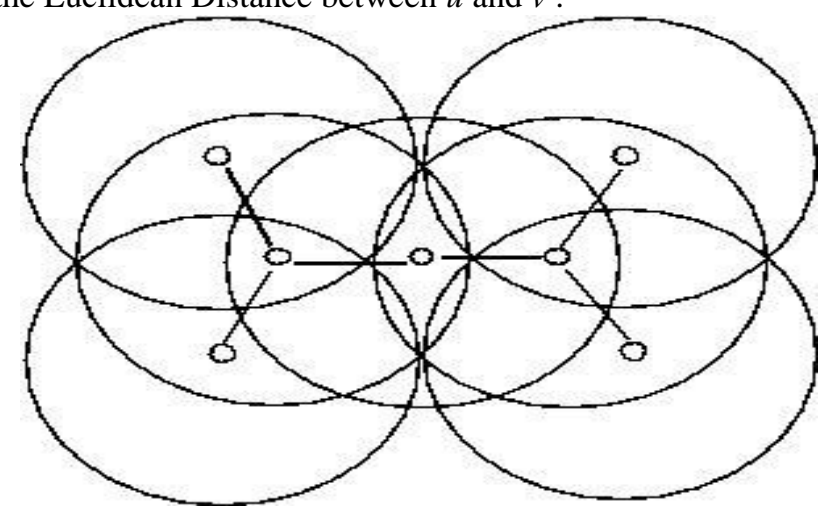

Fig 1: An Unit Disk Graph 


\section{III.ii Dominating Set}

In a graph $\mathrm{G}=(\mathrm{V}, \mathrm{E})$, a subset $\mathrm{C} \subset \mathrm{V}$ is called a dominating set, if all nodes in $\mathrm{G}$ is connected or adjacent to at least one node in C. Simply, we can define a Dominating Set of a graph $\mathrm{G}$ as the set of vertices that covers the entire graph. A Vertex Cover refers to a set of vertices that cover all the edges, whereas a Dominating Set refers to a set of vertices that cover all the vertices. Fig 2 is an example of a dominating set.

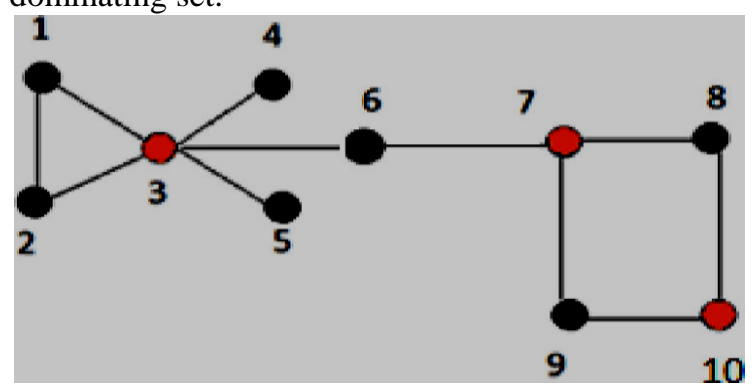

Fig 2: A dominating set $(3,7,10)$

\section{III.iii Connected Dominating Set:}

A connected dominating set CDS of a graph $\mathrm{G}(\mathrm{V}, \mathrm{E})$ is a subset of $\mathrm{V}$ such that there is a path between any node in the CDS . That means a induced connected subgraph is generated.

\section{III.iv Minimum Connected Dominating Set (Mcds):}

In a graph $\mathrm{G}(\mathrm{V}, \mathrm{E})$, there can be more than one connected dominating sets. The connecting dominating set having minimum number of vertices is termed as the minimum connected dominating set

\section{III.v Clustering Coefficient Of A Node}

Clustering is a measure of how "bunched up" (unevenly distributed) the edges of a graph are .Clustering coefficient actually determines the likelihood of any two nodes belonging to the same cluster. The fraction of all pairs of $\mathrm{A}$ 's friends who are also friends Defined only if A has at least two friends (otherwise 0) The clustering coefficient is always between 0 and 1 .

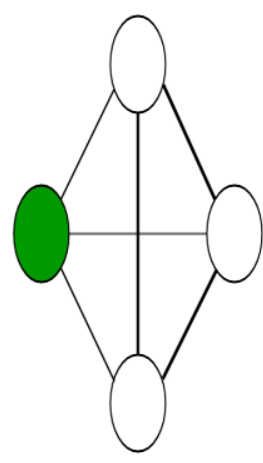

$C=1$

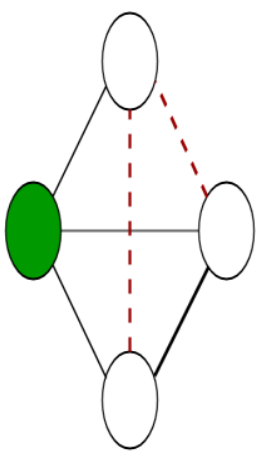

$C=1 / 3$

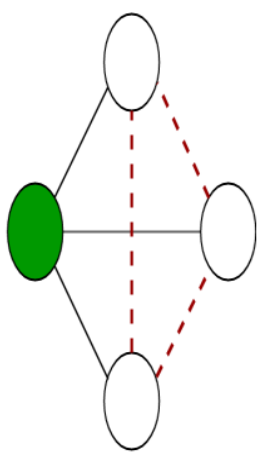

$C=0$
Fig 3: Clustering co efficient of a node

\section{III.vi Clustering Co Efficient Of A Graph}

The clustering coefficient $C C$ of graph $\mathrm{G}$ as shown in fig 4 is the average of the clustering coefficients of all nodes in $G$

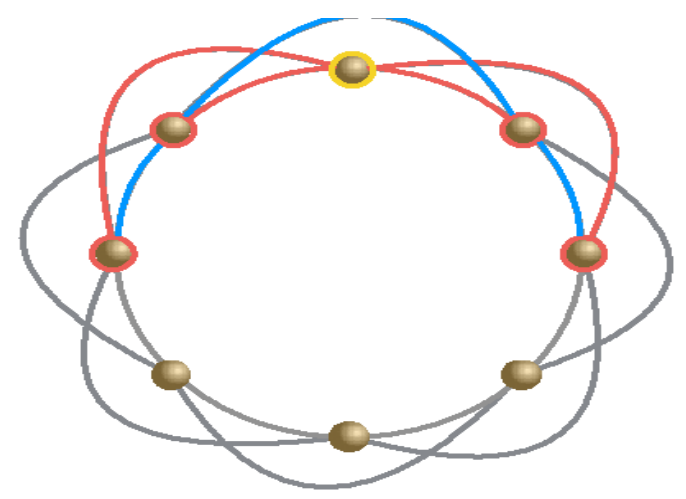

Fig 4: A graph $\mathbf{G}$ with $\mathrm{CC}$

All nodes are identical and have 4 neighbors

Possible edges between pairs of neighbors is $4 \times 3 / 2=6$

How many pairs of neighbors are actually connected? 3

Clustering coefficient of any node: $3 / 6=0.5$

- Clustering coefficient of the entire graph: $C C=0.5$

It is observed that if clustering co efficient of a graph is close to 1 it is highly clustered and if close to 0 then not highly clustered.

\section{PROPOSED METHOD}

In this section, a new method of clustering a mobile adhoc network has been proposed. The new method uses the concept of dominating set.In this method initially we find the dominating set of the mobile adhoc network modelled as a unit disk graph using an algorithm NCDS[12]. Then these dominating nodes are taken as a cluster head of a particular cluster. Then we use the concept of cluster co efficient to find the other member of the cluster .

In graph theory, a clustering coefficient of a vertex in a graph specifies the closeness of the neighboring node that quantifies it to be a complete graph.

The following is the proposed algorithm

DSC-Dominating set Based Cluster

Input: A mobile adhoc network modelled as a cluster Output: A set of cluster

Symbols used in the algorithm

DS- Dominating set

NDS-Non dominating set

$\operatorname{DSC}(\mathrm{G}(\mathrm{V}, \mathrm{E}))$

1. Apply NCDS[12] on $\mathrm{G}(\mathrm{V}, \mathrm{E})$ to find the Connected dominating set DS

2. $\mathrm{N}=|D S|$ i.e number of node in DS

3. For $\mathrm{i}=1$ to $\mathrm{N}$

4. Create $\mathrm{C}_{\mathrm{i}}$ cluster containing a node from DS and make the node a cluster head

5. $\forall x$ in NDS

6. If $x$ is connected to a single dominating node $y$ in DS

7. Include $\mathrm{x}$ in the cluster where $\mathrm{y}$ is the cluster head.

8. Else if $\mathrm{x}$ is connected to more than one node in DS

9. Then Calculate Cluster Stability Index(CSI) of $x$ with respect to the nodes in DS to which $\mathrm{x}$ is connected

10. Include $\mathrm{x}$ in the cluster of the node in DS for which CSI is high.

11. For any cluster $C_{i}$ where $i=1$ to $n$

a. If $|\mathrm{Ci}|=1$

b. Then include it to the cluster to whose cluster head it is directly connected.For mor than 


\section{Dominating Set Based Clustering Algorithm for Mobile Adhoc Network}

one cluster head calculate the CSI and include o the cluster whose cluster head CSI is more.

Cluster Stability Index (x)

$$
\mathrm{CSI}=2 \times \frac{d x}{N x(N x-1)}
$$

Where $\mathrm{dx}$ is the the number of links between the neighbours of $\mathrm{x}$

Ny is the degree of $\mathrm{x}$.

\section{ILLUSTRATION}

Let us implement the proposed algorithm on the following unit disk graph as shown in Fig 5

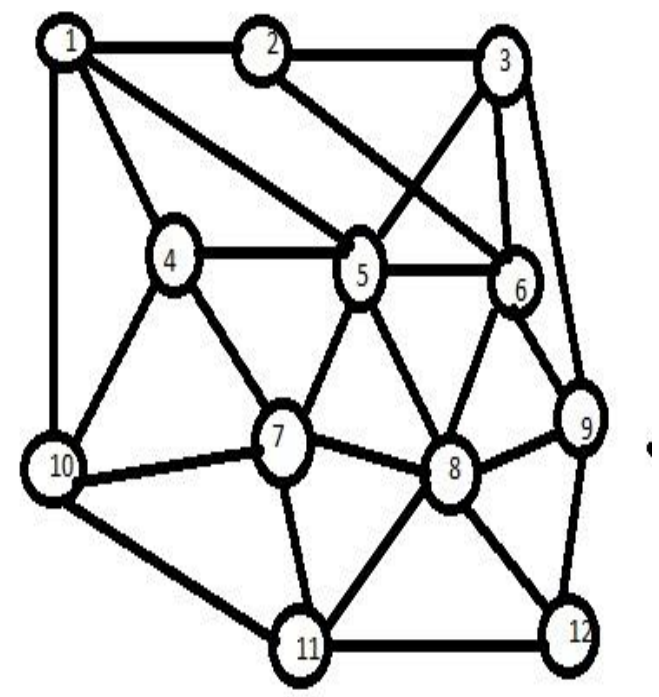

Fig 5: An Unit disk graph

After applying NCDS[12] to the graph in fig 1 we have obtained dominating set

$\mathrm{DS}=\{1,5,3,8\}$. Therefore NDS $=\{2,4,6,7,9,10,11,12\}$

Thus we create 4 clusters each containing one node from DS as cluster head. Now to determine the other member of the cluster we implement the step no 4 to 9 of the algorithm and we get.

Now for node 2

It is connected to 1 and 3 in DS. So we have to find the cluster stability index of both 1 and 2 .

$$
\begin{aligned}
& \operatorname{CSI}(1)=2.1 / 4(4-1)=.16 \\
& \operatorname{CSI}(3)=2.3 / 4(4-1)=.5
\end{aligned}
$$

Since CSI of 3 is more so node 2 is included in the cluster where node 3 is the cluster head.

For node 4

Since node 4 is connected to only one dominating node i.e 1 so it is included in the cluster containing 1 as the cluster head.

\section{For node 6}

Node 6 is connected to dominating node 3,5 and 8

Here CSI $(3)=2.3 / 4(4-1)=.5$

$$
\begin{aligned}
& \operatorname{CSI}(5)=2.3 / 5(5-1)=0.3 \\
& \operatorname{CSI}(8)=2.6 / 6(6-1)=0.4
\end{aligned}
$$

Here CSI of 3 is high among all the three node so node 6 is included in the cluster of node 3

\section{For node 7}

Node 7 is connected to dominating node 5 and 6 .

Here CSI $(5)=2.3 / 5(5-1)=0.3$

$$
\operatorname{CSI}(8)=2.6 / 6(6-1)=0.4
$$

Here CSI of node 8 is more thus node 7 is included in the cluster of node 8 .

For node 9

Node 9 is connected to node 3 and 8

Here CSI of node 3 is more so it is included in the cluster of node 3 .

Fore node 10

Node 10 is connected to only one dominating node i.e.1. so it is included in the cluster of node 1

\section{For node 11}

Node 11 is connected to only one dominating node i.e node 8 so it is included in the cluster containing node 8.

\section{For node 12}

Node 12 is connected to only one dominating node i.e 8 so it is included in the cluster of node 8 .

Thus we get the following clusters as shown in Fig 6
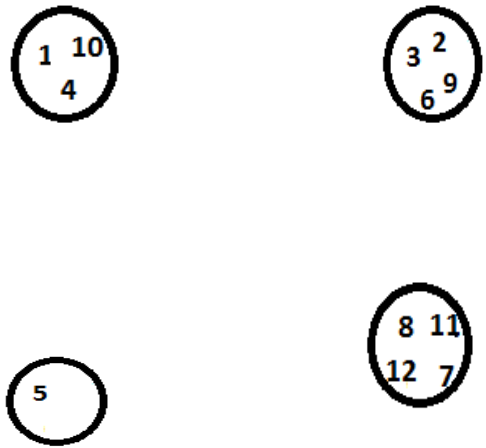

Fig 6: Individual cluster

It is observed that four clusters are formed and and one cluster contain only the cluster head. So this cluster will be merged to one of the remaining clusters according to step number 11 of the proposed algorithm. Since node 5 is connected to two cluster head namely node 3 and 8 so CSI of node 3 and 8 is compared . Here CSI(3) $>\operatorname{CSI}(8)$ and hence node 5 is included in the cluster of node 3 . Thus we obtain the following cluster (Fig 7) of the above unit disk graph (Fig 5).

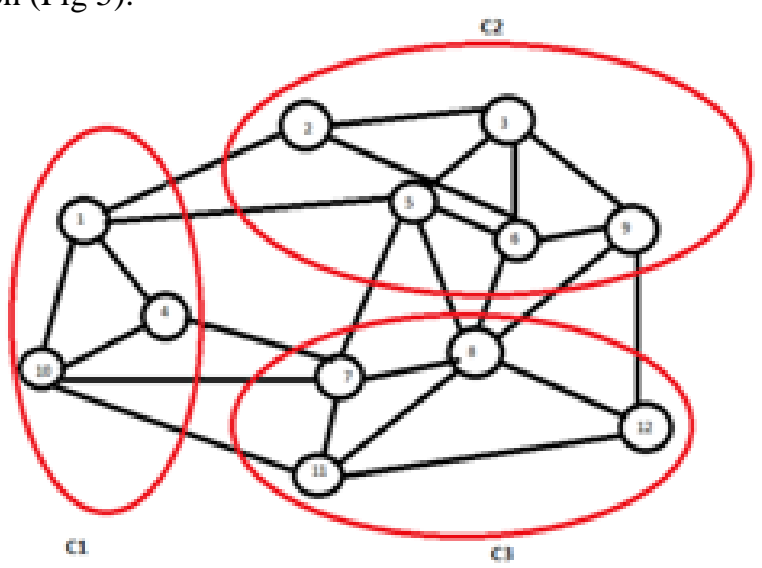

Fig 7: Resultant Cluster 


\section{PROPOSED METHOD FOR SELECTING THE GATEWAY NODE}

After creating the cluster we need to select the gateway node for inter cluster communication. The gateway node should be selected in such a way that it can easily relay packets from one cluster to another. Since the gateway node is going to forward packets to cluster head of another cluster so while selecting the gateway node the cluster head nodes should be taken into consideration. Here we use the concept of hop count to calculate the distance between an ordinary node and cluster head node.The main idea is to calculate the distance between ordinary nodes of one cluster and the cluster head node of another cluster. The nodes having the minimum distance is selected as the cluster gateway node. This is repeated for any two clusters. In a cluster, the selection of the gateway node of the source as well as destination cluster is done on the basis of minimum distance from the cluster head of the respective cluster that reduces energy consumtion in the entire network.Using this method,initially for each node in the source as well as destination cluster, it's distance from the cluster head is calculated and the node having the minimum distance from the respective cluster head is selected as the gateway node.

The algorithmic steps are given below.

\section{Symbols used in the algorithm:}

C1- cluster 1

C2- cluster 2

$\mathrm{V}(\mathrm{C} 1)=$ set containing the nodes in $\mathrm{C} 1$

$\mathrm{V}(\mathrm{C} 2)=$ set containing the nodes in $\mathrm{C} 2$

\section{GatewayNodeSelction( $\mathrm{C} 1$ and $\mathrm{C2})$}

1. For any two cluster $\mathrm{C} 1$ and $\mathrm{C} 2$

2. Let $\mathrm{x}$ is the cluster head of $\mathrm{C} 1$ and $\mathrm{y}$ is the cluster head of $\mathrm{C} 2$

3. Set $\mathrm{V}^{\prime}(\mathrm{c} 1)=\mathrm{V}(\mathrm{C} 1)-\mathrm{x}$

4. Set $\mathrm{V}^{\prime}(\mathrm{c} 2)=\mathrm{V}(\mathrm{C} 2)-\mathrm{y}$

5. For all $\mathrm{v}$ in $\mathrm{V}^{\prime}(\mathrm{c} 1)$ and all $\mathrm{u}$ in $\mathrm{V}^{\prime}(\mathrm{c} 2)$

6. Calculate distance between $\mathrm{v}$ and $\mathrm{y}$ and distance between $\mathrm{u}$ and $\mathrm{x}$

7. Select the node in $\mathrm{V}^{\prime}(\mathrm{c} 1)$ and $\mathrm{V}^{\prime}(\mathrm{c} 2)$ as the gateway node of respective cluster whose distance is minimum from $\mathrm{x}$ and $\mathrm{y}$

In our example for cluster $\mathrm{C} 1$ and $\mathrm{C} 2$

$\mathrm{V}(\mathrm{C} 1)=\{1,4,10\}$

$\mathrm{V}(\mathrm{C} 2)=\{2,3,5,6,9\}$

Here 1 is the cluster head of $\mathrm{C} 1$ and 3 is the cluster head of $\mathrm{C} 2$

Now for cluster $\mathrm{C} 1$ its ordinary nodes distance from the cluster head node 3 of cluster 2 is calculated as shown in Table 2

\begin{tabular}{|l|l|l|}
\hline $\begin{array}{l}\text { Ordinary } \\
\text { node }(\mathrm{o})\end{array}$ & $\begin{array}{l}\text { Cluster } \\
\text { head(h) }\end{array}$ & Dist $(\mathrm{o}$ and $\mathrm{h})$ \\
\hline 4 & 3 & 3 \\
\hline 10 & 3 & 3 \\
\hline
\end{tabular}

\begin{tabular}{|l|l|l|}
\hline & & \\
\hline & & \\
\hline & & \\
\hline
\end{tabular}

Table 2
In this case both the nodes are at same minimum distance from the cluster head so arbitrarily we can select any one as the cluster head. Let us select node 4 as the cluster head of cluster 1

Similarly for cluster 2 its ordinary nodes distance from the cluster head node 1 of cluster 1 is calculated as shown in Table 3

\begin{tabular}{|l|l|l|}
\hline $\begin{array}{c}\text { Ordinary } \\
\text { node }(\mathrm{o})\end{array}$ & Cluster head(h) & Dist(o and $\mathrm{h})$ \\
\hline 2 & 1 & 1 \\
\hline 5 & 1 & 1 \\
\hline 6 & 1 & 2 \\
\hline 9 & 1 & 3 \\
\hline
\end{tabular}

Table 3

Here node 2 and 5 are at equal distance from the cluster head of $\mathrm{C} 1$, so arbitrarily we select node 2 as the gateway node of cluster 2.Similary for other cluster also the gateway nodes are selected as per the above procedure

\section{CONCLUSION}

Clustering is one of the important research area in MANET. This paper explores the fundamental concepts of clustering and reviewed the various existing clustering algorithm in MANET. The proposed new clustering algorithm is based on the concept of dominating set. Also we have proposed another method for selecting gateway node of a cluster. In future, the quality of cluster formed by the proposed method will be discussed

\section{REFERENCES}

1. M. Gerla, J. T. C. Tsai, "Multicluster, mobile, multimedia radio network," ACM/Baltzer Wireless Networks Journal 95, vol. 1, Oct. 1995 , pp. $255-265$

2. A. Ephremides, J. E. Wieselthier,D. J. Baker, "A design concept for reliable mobile radio networks with frequency hopping signaling," Proceedings of the IEEE, vol. 75, no. 1, Jan. 1987, pp $56-73$

3. D. J. Baker, A. Ephremides, "The architectural organization of a mobile radio network via a distributed algorithm," IEEE Trans. on Comm., vol. COM-29, pp.

4. S. Basagni, "Distributed clustering algorithm for ad-hoc networks,' In International Symposium on Parallel

Architectures, Algorithms, and Networks (I-SPAN), pp.310-315, 1999.

5. S. Basagni, "Distributed and mobility-adaptive clustering for multimedia support in multi-hop wireless networks," Proceedings of Vehicular Technology Conference, VTC, vol. 2, fall 1999, pp. 889 893.

6. X. Niu, Z. Tao, G. Wu, C. Huang, L. Cui, "Hybrid cluster routing. an efficient routing protocol for mobile ad hoc networks," IEEE ICC 2006

7. J.Y. Yu, P. H. Joo Chong, "An efficient clustering scheme for large and dense mobile ad hoc networks

(MANETs)," Computer Communications No .30, 2006,pp. 5-16

8. M. Chatterjee, S. K. Das, and D. Turgut, "WCA: A weighted clustering algorithm for mobile ad hoc

networks," Journal of Cluster Computing, No. 5, 2002, pp. 193-204.

9. Z. El-Bazzal, M. Kadoch, B. L. Agba, F. Gagnon and M. Bennani, "An efficient management algorithm for

clustering in mobile ad hoc network," ACM. October 2, 2006, Torremolinos, Malaga, Spain.

10. E. Qaddoura,W .AMobaideen and A. Omari "Distributed clusterhead architecture for mobile ad hoc networks,"

Journal of Computer Science, No 2(7), pp. 583-588 


\section{Dominating Set Based Clustering Algorithm for Mobile Adhoc Network}

11. The Network Simulator - ns-2 http://www.isi.edu/nsnam/ns/ 381

12. B. Kalita, A. K. Das, A.U.Islam, Power Efficient Routing in Mobile Adhoc Network (MANET) Using Connected Dominating Set, International Journal of Computer Sciences and Engineering, Vol.-6, Issue-10, Oct 2018 E-ISSN: 2347-2693

\section{AUTHOR PROFILE}

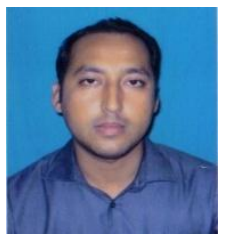

Arnab Kumar Das is presently working as an Assistant Professor in the Department of Computer Application, GIMT Guwahati and is also a research scholar under faculty of Engineering, Gauhati University, Gauhati, Assam. He has completed his Master degree (MCA) from Dibrugarh University in 2007 and M Tech (IT) from Gauhati University in 2014. His research interest is Graph theory, Algorithms and Network. He has 10 years of teaching experience.

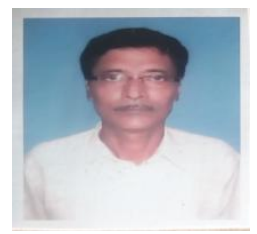

Bichitra Kalita , retired HOD and Associate professor, Assam Engineering College, Guwahati, now working as Professor, Dept of Computer Applications, Assam Don Bosco University, Azara, Guwahati, Assam. He has altogether published 56 research paper in national and international journals in various fields of mathematical and computer science. He supervised 9 scholars who have obtained their Phd degree from Gauhati Univesity in different fields. He used graph theoretic techniques to solve the long standing Goldbach conjecture in 2013. His research interest is graph theory, NP-complete problems, Graph Data base etc. 